\title{
XXI.
}

\section{Kleinere Mittheilungen.}

\section{Zur Statistik der Amputationen.}

\author{
Von
}

Prof. Dr. V. v. Bruns.

In dem dritten Hefte des vorliegenden Bandes dieser Zeitschrift S. 334 schreibt Herr Professor Dr. Hu eter in Greifswald bei Besprechung meiner Schrift: "Die Amputation der Gliedmassen durch Zirkelschnitt mit vorderem Hautlappen. Tübingen 1879" u. A.: "Bemerkenswerth ist, dass , unter den hier einbegriffenen 30 Oberschenkelamputationen nur 3 tödtlich "endeten."

Ich bin jetzt nach Ablauf eines weiteren Jahres in der glücklichen Lage, das von Hueter hervorgehobene günstige Ergebniss meiner Oberschenkelamputationen durch eine weitere Reihe von Amputationsfällen vervollständigen zu können, durch welche sich das angegebene Verhältniss noch günstiger gestaltet hat. In dem Laufe des Jahres 1879 habe ich nämlich 9 Amputationen des Oberschenkels gemacht, ohne einen einzigen Todesfall darunter beklagen zu müssen, wodurch die Zahl der in den letzten füf Jahren in meiner Klinik ausgeführten einfachen Oberschenkelamputationen auf die Zahl von 39 mit nur 3 Todesfällen gestiegen ist.

Zur vollständigeren Orientirung des Lesers, dem meine Eingangs erwähnte Schrift über Amputation nicht zur Hand ist, mag es mir gestattet sein, die am Schlusse derselben gegebene kleine Tabelle über die in meiner Klinik in den letzten vier Jahren - 1875 bis 1878 - gemachten grossen Amputationen hier wieder zu geben, und zwar vermehrt mit den in dem eben abgelaufenen Jahre - 1879 - vorgekommenen $25 \mathrm{Ampu}$ tationen, welche insgesammt einen giunstigen Erfolg genommen haben.

Amputationen der grossen Gliedmassen in der chirurgischen Klinik in Tübingen in den Jahren 1875-1879.

\begin{tabular}{|c|c|c|c|c|c|c|c|c|c|c|}
\hline & \multicolumn{2}{|c|}{ Oberarm } & \multicolumn{2}{|c|}{ Vorderarn } & \multicolumn{2}{|c|}{ Oberschenkel } & \multicolumn{2}{|c|}{ Unterschenkel } & \multicolumn{2}{|c|}{ Summa } \\
\hline & $\underset{\text { zahl }}{\text { Gesanmt- }}$ & $\left|\begin{array}{c}\text { davon } \\
+\end{array}\right|$ & $\underset{\text { zahl }}{\text { Gesammt- }}$ & $\left|\begin{array}{c}\text { davon } \\
+\end{array}\right|$ & $\underset{\text { zahl }}{\text { Gesammt- }}$ & $\stackrel{\text { davon }}{+}$ & ${ }_{\text {zahl }}^{\text {Gesammt- }}$ & $\mid \begin{array}{c}\text { davon } \\
+\end{array}$ & $\underset{\text { zahl }}{\text { Gesammt- }}$ & $\mid \begin{array}{c}\text { davou } \\
+\end{array}$ \\
\hline 1875 & 2 & - & - & - & 4 & - & 10 & 2 & 16 & 2 \\
\hline 1576 & 2 & 1 & 1 & - & 8 & 1 & 4 & - & 15 & 2 \\
\hline 1877 & 2 & - & 2 & - & 3 & - & 11 & 4 & 18 & 4 \\
\hline 1878 & 1 & - & 2 & 1 & 15 & 2 & 8 & 1 & 26 & 4 \\
\hline 1879 & 1 & - & 2 & - & 9 & - & 13 & - & 25 & - \\
\hline $875 / 79$ & 8 & 1 & 7 & 1 & 39 & 3 & 46 & 7 & 100 & 12 \\
\hline
\end{tabular}


Der vorstehenden Tabelle habe ich noch folgende erläuternde Bemerkungen beizufïgen.

1. Ausgeschlossen aus dieser Tabelle sind die Doppelamputationen, wie diese auch von R. Volkmann, Schede u. A. von den einfachen Amputationen und zwar mit Recht getrennt werden. In dem abgelaufenen Jahre 1879 sind 4 Doppelamputationen in meiner Klinik gemacht worden und zwar sämmtlich mit tödtlichem Ausgange, nämlich

1 Amputation beider Oberschenkel wegen Zermalmung beider Unterschenkel durch Ueberfahren auf der Eisenbahn. Tod an Shok nach 4 Stunden.

1 Amputation des rechten Oberschenkels und des linken Unterschenkels hoch oben wegen der gleichen Verletzung. Tod an Trismus nach 14 Tagen günstigen Verlaufs.

2 Amputationen beider Unterschenkel, davon die eine wegen spontaner Gangrän beider Füisse und die andere wegen umfangreicher Verschwärung beider Füsse mit hochgradiger Klumpfussstellung. Beide starben an Tetanus.

2. Dagegen ist unter die 9 Oberschenkelamputationen des Jahres 1879 eine Exarticulation des Oberschenkels aus dem Hüftgelenke aufgenommen, welche ich in der Weise ausgefuhrt habe, dass nach vorangeschickter Unterbindung der Arteria cruralis dicht unter dem Ligamentum Poupartii ein grosser vorderer Hautlappen gebildet, dann die uhrigen Weichtheile durch Zirkelschnitt getrennt, der Knochen in der Trochanterpartie quer durchsägt und schliesslich der Schenkelkopf nebst Hals aus der Pfanne mit Messer und Pincette ansgelöst wurde. Es ist dieses Verfahren der Auslösung des Schenkels aus der Hüftpfanne im Wesentlichen das gleiche Verfahren, welches ich bei meiner ersten derartigen Operation im Juli 1844 befolgt und 1847 veröffentlicht habe 1). Die im Jahre 1844 wegen eines grossen Osteosarkoms Operirte ward nach nahezu 5 Monaten, der 1879 wegen multipler Sarkombildung in dem Oberschenkelknochen Operirte nach 5 Wochen vollständig geheilt aus der Klinik entlassen.

3. Die Todesursachen der in der Tabelle aufgeführten 12 Fälle mit tödtlichem Ende sind folgende nach den Jahren angeordnet:

1875 Erschöpfung bei einer Amputatio cruris, Pyämie bei einer Amputatio cruris,

1876 Pyämie bei einer Amputatio humeri, Pyämie bei einer Ampntatio femoris,

1877 Erschöpfung bei einer Amputatio cruris, Gehirnerweichung bei einer Amputatio cruris, Pyämie bei einer Amputatio cruris, Erschöpfung bei einer Amputatio cruris,

1878 Erschöpfung durch Diarrhoe bei einer Amput. femoris,

1) Uebersicht der in der chirurgischen Klinik zu Tübingen vom Frühjahr 1843 bis Spätjahr 1846 vorgekommenen Krankheitsfälle und Operationen nebst Mittheilung einer Auslösung des Oberschenkels aus dem Hüftgelenke von Victor Bruns. Mit 3 Abbildungen. Tübingen 1847. $4^{\circ}$. S. 19. 
1878 Pyämie bei einer Amputatio cruris, Herzfehler u. Thrombose bei einer Amput. femoris, Fettherz, Morb. Addisonii bei einer Amp. antibrachii.

4. Das Jahr 1879 ist das erste, in welchem kein einziger Todesfall an Pyämie oder Septicämie in meiner Klinik vorgekommen ist, weder nach Operationen noch bei sonstigen Erkrankungen.

Die Gesammtzahl der in diesem Jahre in der Klinik behandelten Kranken beträgt 600 ; die Zahl der vorgenommenen grösseren blutigen Operationen beziffert sich auf mehr denn 200 .

5. Operation und Nachbehandlung bei allen Amputationen sind, wie ich in meiner Eingangs erwähnten Schrift über Amputation näler erörtert habe, streng nach antiseptischen Grundsätzen ausgeführt worden, nur mit der Abweichung, dass in den früheren Jahren in einzelnen Fällen, seit dem Frühjahr 1879 aber durchgehends der Carbolspray bei den Operationen und bei dem Verbande grundsätzlich weggelassen und durch temporäre Berieselung des Operationsfeldes mit 2-3 procentigem Carbolwasser aus einem gläsernen Heberirrigator ersetzt worden ist. Näheres hierüber sowie über die sonstigen Vereinfachungen des antiseptischen Verbandapparates in meiner Klinik behalte ich mir für einen andern Ort vor. 

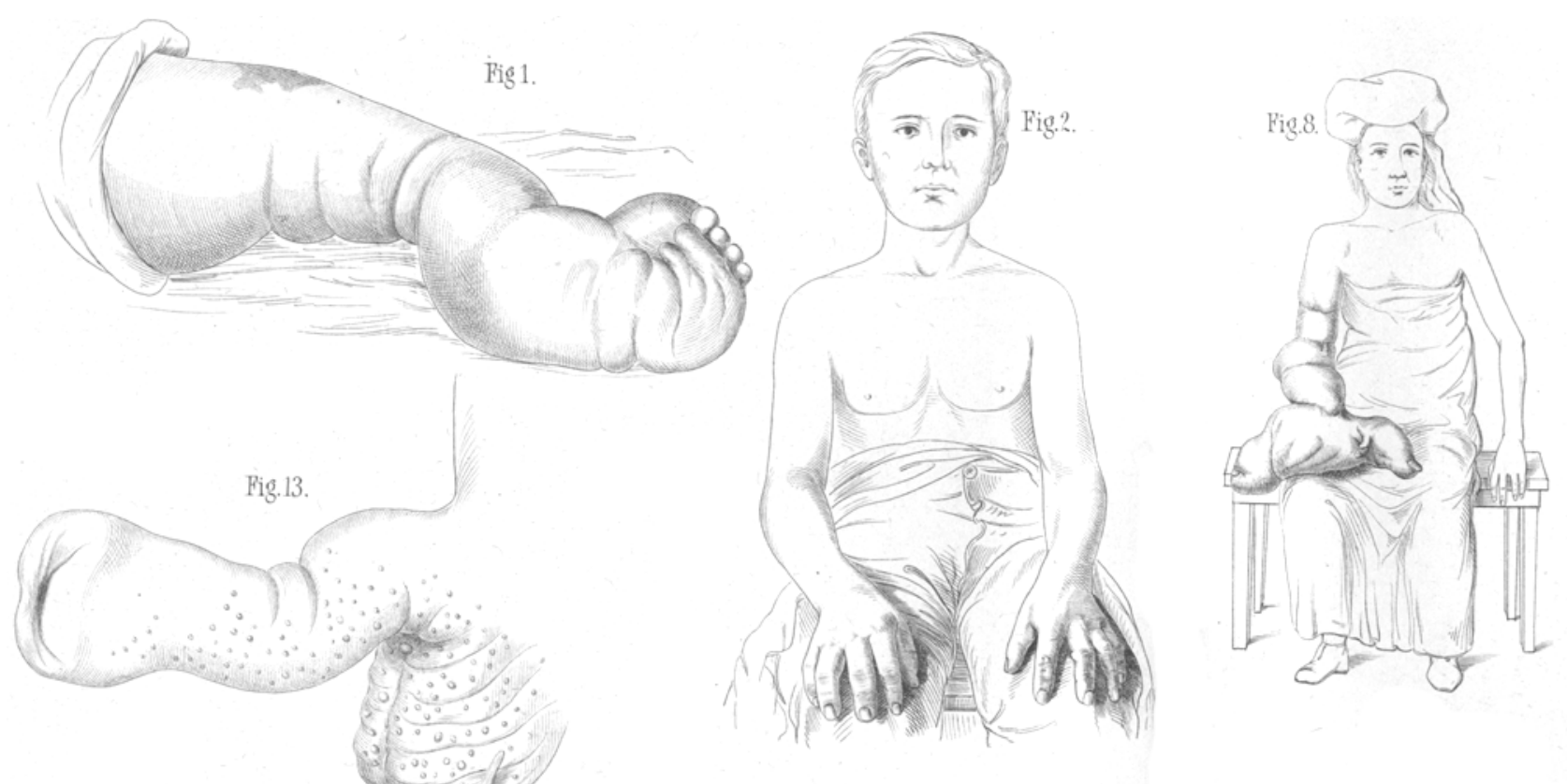

Fig.12.

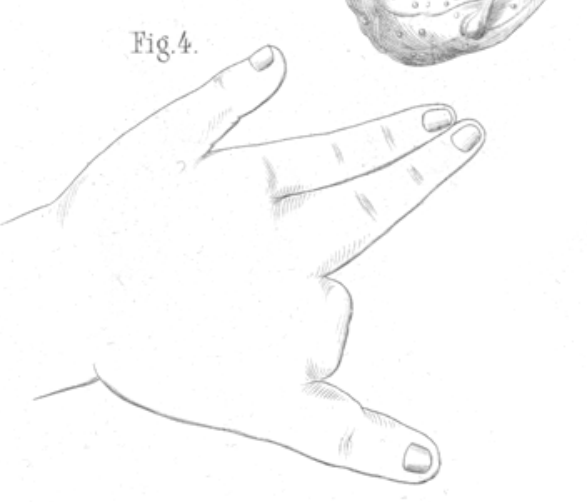

Fig.7.
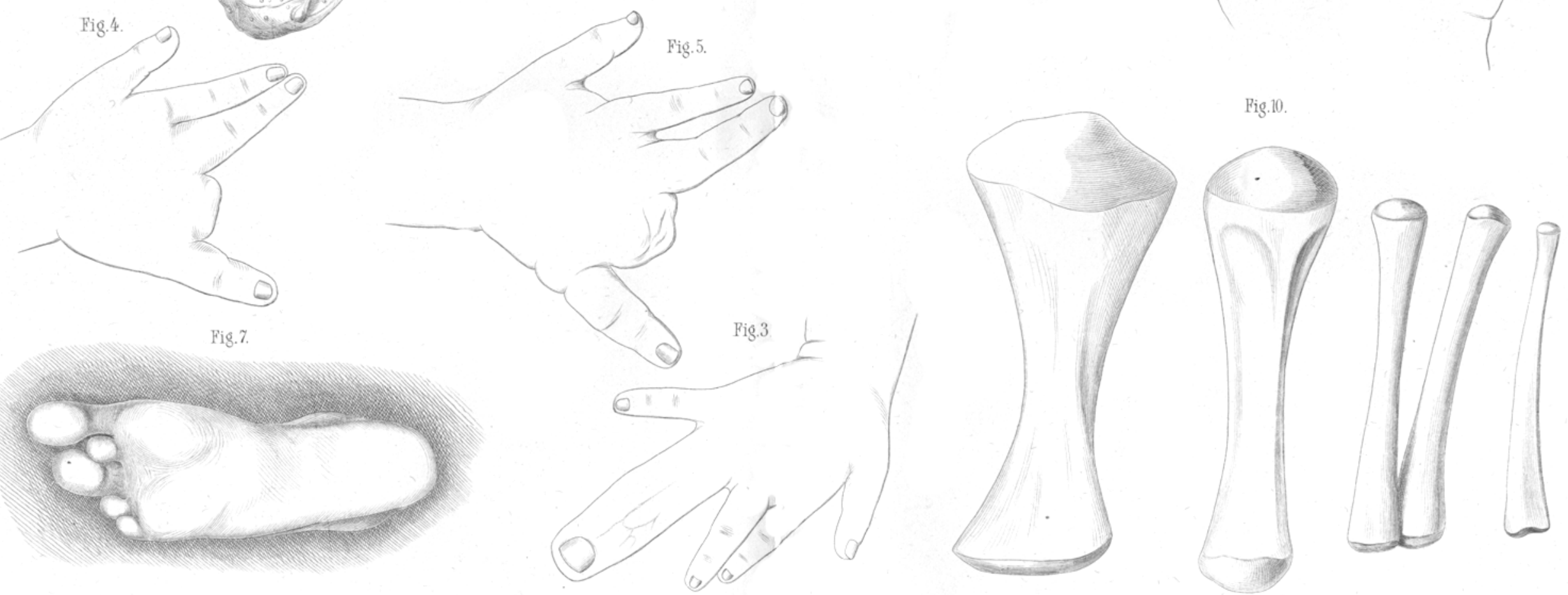


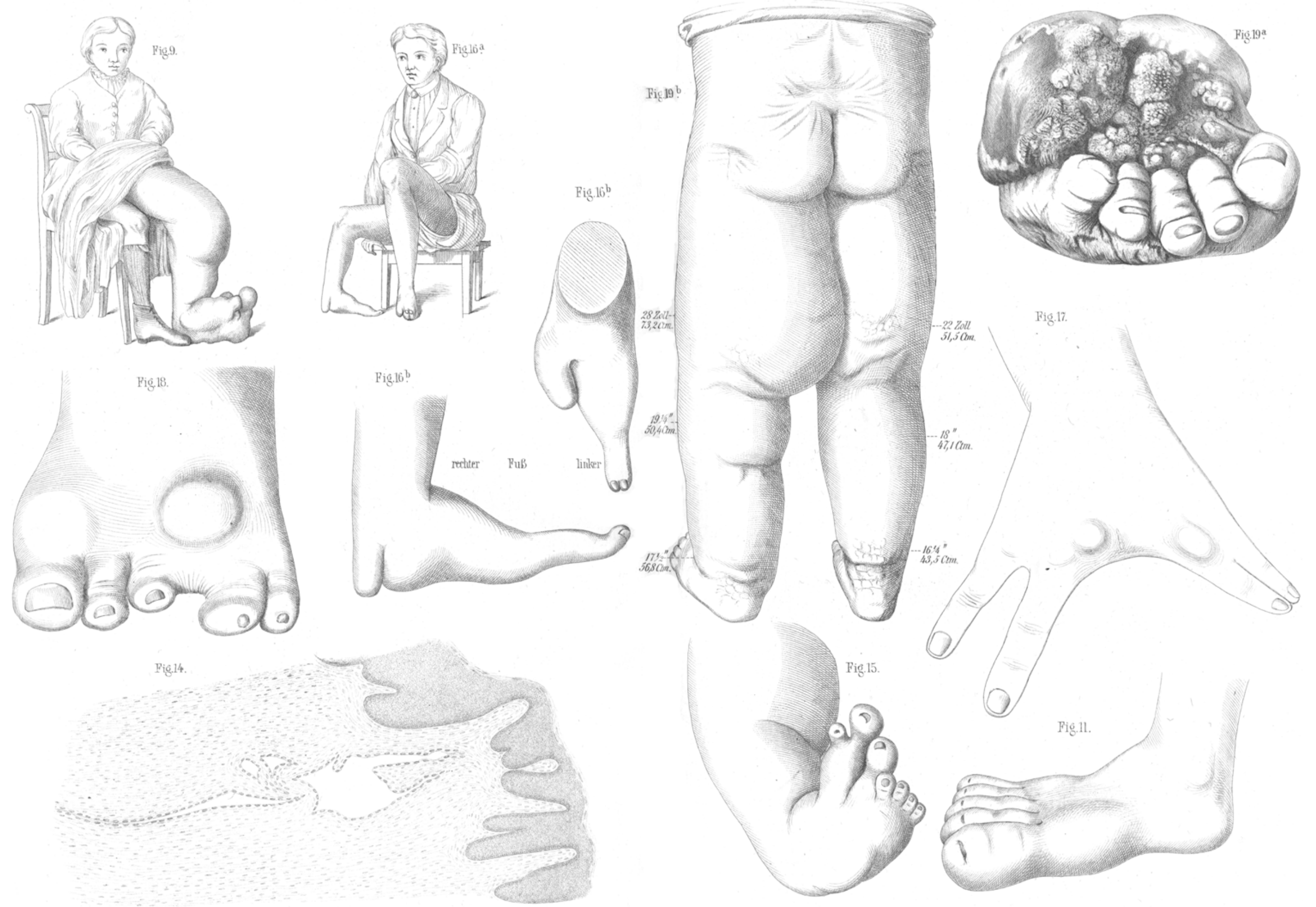


Deuts she Zeitschrifft f. Chirurgie.Bd.XII.

Tafel V.

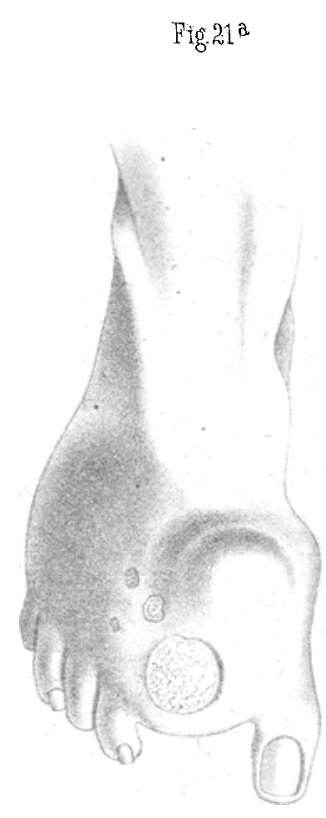

Fig. 21 b

Fig. 20

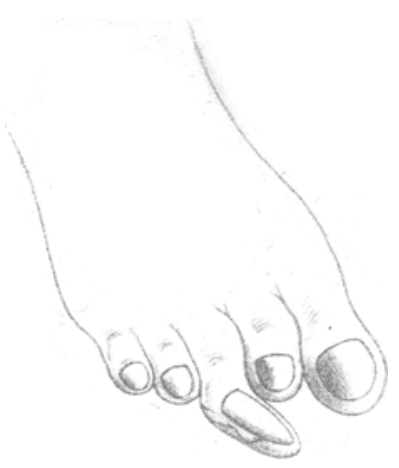

$1 / 3$ nat br. 

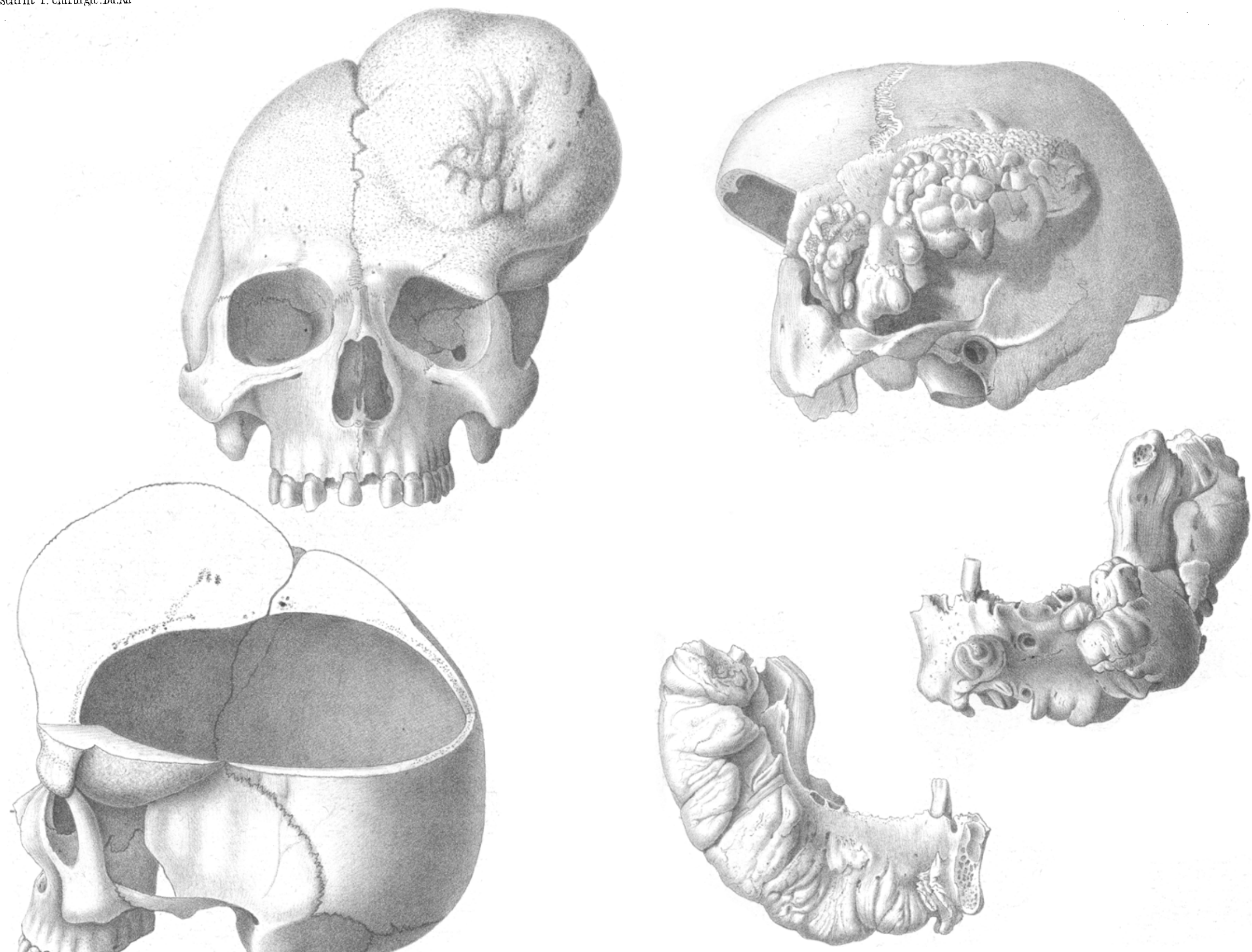

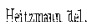



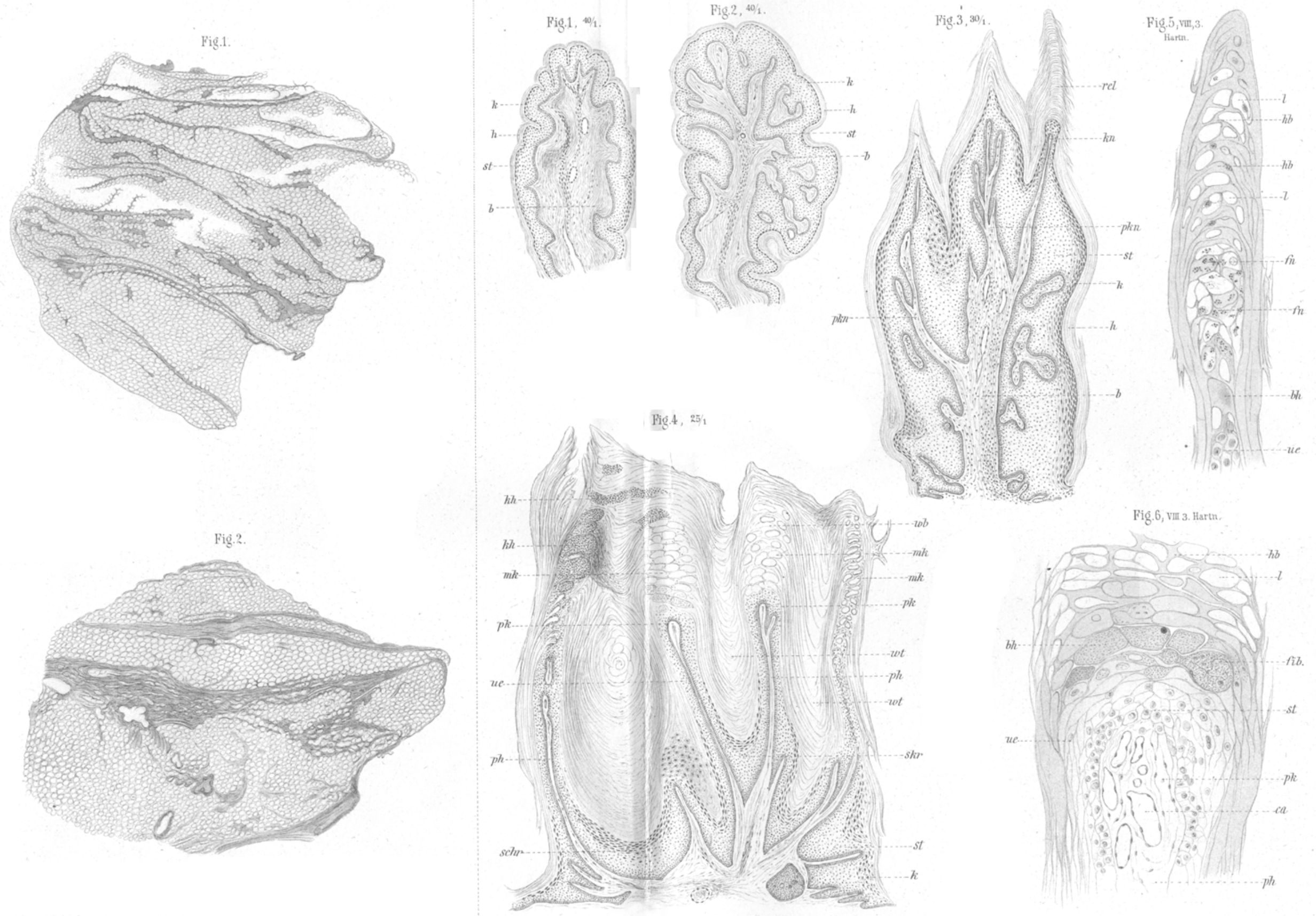

Hatuman 2

Unma, Fibrokeratom

tilas von F.c.r. Vogel in Lépzig

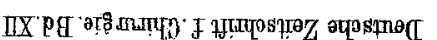




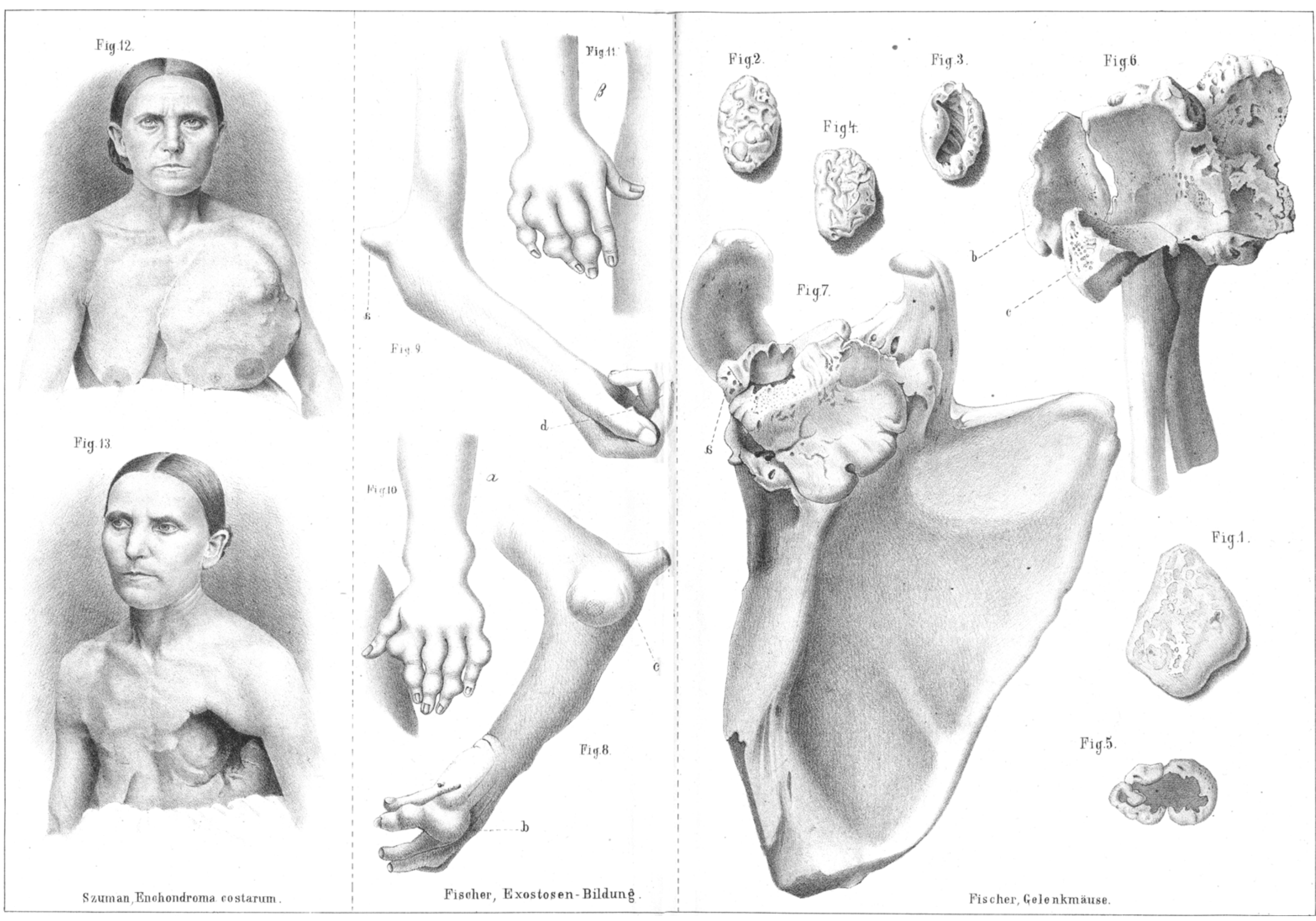


